\title{
Impact of Road Vehicle Accelerations on SAR-GMTI Motion Parameter Estimation
}

\author{
Stefan Baumgartner*, Martina Gabele*, Gerhard Krieger", \\ Karl-Heinz Bethke*, Sergey Zuev \\ *Microwaves and Radar Institute, German Aerospace Center (DLR) \\ Muenchner Straße 20, 82234 Weßling, GERMANY \\ email: Stefan.Baumgartner@dlr.de
}

\begin{abstract}
In this paper the problem of reliable along-track velocity estimation is addressed. It is known that the neglection of across-track accelerations can bias the estimates of along-track velocities, but up to now it is unknown which vehicle accelerations appear in real traffic scenarios. On this account an experiment to measure accelerations was conducted. The acceleration measurement results, which are presented and discussed in this paper, imply that accelerations must not be neglected in the alongtrack velocity estimation step if accurate estimates are required. Finally, some basic ideas are given which enable a reliable separation of along-track velocity and acrosstrack acceleration and so improve the estimation accuracy.
\end{abstract}

\section{Introduction}

In recent years many powerful techniques and algorithms have been developed to detect moving targets and to estimate their motion parameters from single- or two-channel SAR data. Most of these

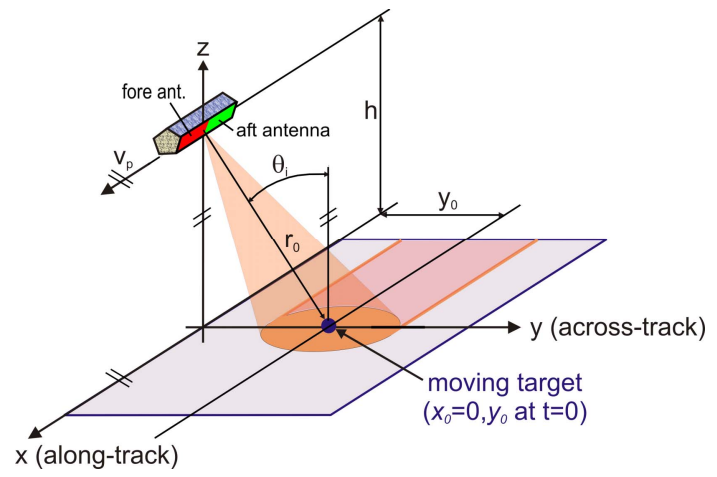

Figure 1: Two-channel side-looking radar geometry. algorithms rely more or less on the analysis of the Doppler history of the moving target signal. Commonly the along-track velocity is calculated by using the estimated Doppler slope under the implicit assumption that the across-track acceleration is very small and therefore negligible. In [1] it is shown that even small across-track accelerations can bias the along-track velocity estimation. Since we want to monitor complex traffic scenarios with a future traffic monitoring system like TRAMRAD [2], we must know with which vehicle accelerations we have to deal in reality and with which estimation errors we have to cope with if these accelerations are neglected.

For this reason an experiment to measure accelerations of a common passenger car during rush hour traffic on major roads and autobahns was conducted. Before the acceleration measurement results are presented and discussed, the problem of reliable along-track velocity estimation will be recapitulated.

\section{Along-Track Velocity Estimation Error}

For the following investigations the Cartesian radar geometry shown in Figure 1 is considered. The instantaneous motion parameters of the moving point target at broadside time $t=0$, where the target is located at $\left(x=0, y=y_{0}, z=0\right)$, are: along-track velocity $v_{x 0}$, across-track velocity $v_{y 0}$, along-track acceleration $a_{x 0}$ and across-track acceleration $a_{y 0}\left(a_{x 0}\right.$ and $a_{y 0}$ remain constant during the whole observation time). The target doesn't move in z-direction.

Assuming that nowadays it is principally possible to estimate the across-track velocity $v_{y 0}$ of a moving vehicle and additionally the Doppler slope $k_{a}$ of the corresponding signal with high accuracy, there still remains the problem of reliable along-track velocity estimation. Following the derivation in [1] and also ignoring across-track acceleration and velocity, the along-track velocity can be computed by 
exploiting the estimated Doppler slope $\hat{k}_{a}$ in the following way (it is assumed that the Doppler slope can be estimated exactly, i.e. $\hat{k}_{a} \equiv k_{a}$ ):

$$
\hat{v}_{x 0}=v_{p}-\sqrt{-\frac{1}{2} \hat{k}_{a} \lambda r_{0}} \stackrel{\hat{k}_{a} \equiv k_{a}}{\longrightarrow} \quad \hat{v}_{x 0}=v_{p}-\sqrt{\left(v_{x 0}-v_{p}\right)^{2}+v_{y 0}^{2}\left(1-\frac{y_{0}^{2}}{r_{0}^{2}}\right)+y_{0} a_{y 0}},
$$

where $v_{p}$ is the SAR platform velocity in along-track direction, $\lambda$ the wavelength of the carrier frequency and $r_{0}$ the distance to the target at broadside time $t=0$. The along-track velocity error $\Delta v_{x 0}$ or bias, respectively, is then given by $\Delta v_{x 0}=\hat{v}_{x 0}-v_{x 0}$. It can be shown that for SAR systems where $v_{p}>>v_{x 0}$ and $v_{p}>>v_{y 0}$ the following simple approximation describing the along-track velocity estimation error can be used:

$$
\Delta v_{x 0} \cong-\frac{y_{0}}{2 v_{p}} a_{y 0}=-\frac{r_{0} \sin \theta_{i}}{2 v_{p}} a_{y 0} .
$$

Considering a SAR system operating in low-earth-orbit (LEO, Cartesian geometry shown in Figure 1 assumed) with $v_{p}=7300 \mathrm{~m} / \mathrm{s}$ and $y_{0}=514 \mathrm{~km}$, a constant across-track acceleration of $0.25 \mathrm{~m} / \mathrm{s}^{2}$ causes an along-track velocity estimation error of about $-32 \mathrm{~km} / \mathrm{h}$. Such high along-track velocity estimation errors are not tolerable for traffic monitoring systems.

\section{Acceleration Measurements}

To find out which vehicle accelerations occur in complex traffic scenarios generally, an experiment to measure accelerations was conducted in cooperation with the DLR Institute of Transport Research in

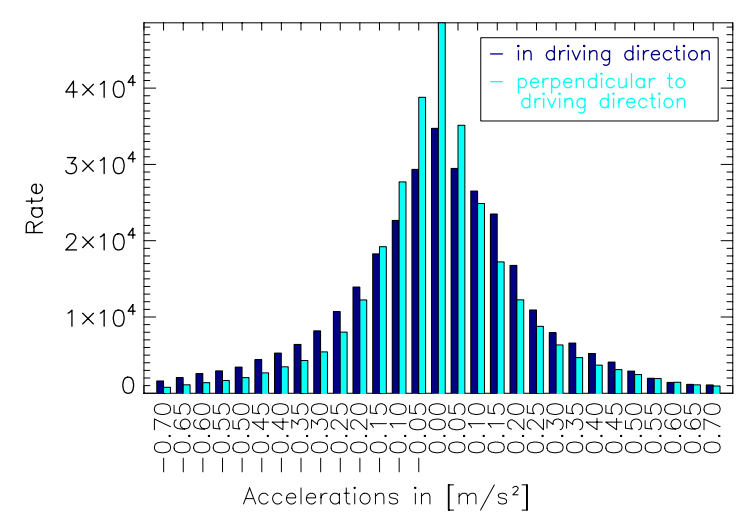

Figure 2: Histogram of measured accelerations. Berlin in July 2005. For measuring the acceleration components in all three spatial dimensions a common passenger car has been equipped with a calibrated inertial measurement system (IMU) and DGPS. Data were acquired half an hour long with a sampling frequency of $200 \mathrm{~Hz}$. The histogram of the measured passenger car horizontal accelerations (moving average filtered to decrease the influence of sensor noise, window width $0.055 \mathrm{~s}$ ) is shown in Figure 2. The determined standard deviation of the acceleration component in driving direction was 0.45 $\mathrm{m} / \mathrm{s}^{2}$ and perpendicular to driving direction $0.61 \mathrm{~m} / \mathrm{s}^{2}$. Alone the magnitudes of the standard deviations and hence the large expected along-track velocity estimation error imply that across-track accelerations must not be neglected in the along-track velocity estimation step. The minimum and maximum values of the measured accelerations are of less interest since their occurrence in the measurement data is temporally limited [4].

To see the influence of real measured accelerations on SAR imagery and to make more reliable statements regarding along-track velocity estimation errors, simulations have been performed. Therefore, the Cartesian geometry shown in Figure 1 with $r_{0}=729 \mathrm{~km}, \theta_{i}=45^{\circ}$ and $v_{p}=7300 \mathrm{~m} / \mathrm{s}$ as well as a synthetic aperture time $T_{S A}=0.64 \mathrm{~s}$ (e.g. LEO system) were used. The time varying position of the point target in the $\mathrm{x}-\mathrm{y}$ plane was computed by using the original measured (not filtered) acceleration values. With a filter bank matched to different along-track velocities or quadratic phase errors, respectively, the along-track velocity errors have been estimated (Figure 3). We found that the velocity errors estimated by matched filter banks are in good agreement with the errors computed by using equation (2) with average acceleration values arising during the synthetic aperture time. Deviations of both errors from each other (Figure 3, lower right) occur mainly due to uncompensated higher order phase terms which arise e.g. by fast time varying accelerations. Especially at longer synthetic aperture times in the order of several seconds (e.g. at airborne systems), these higher order phase errors might become more and more dominant. 


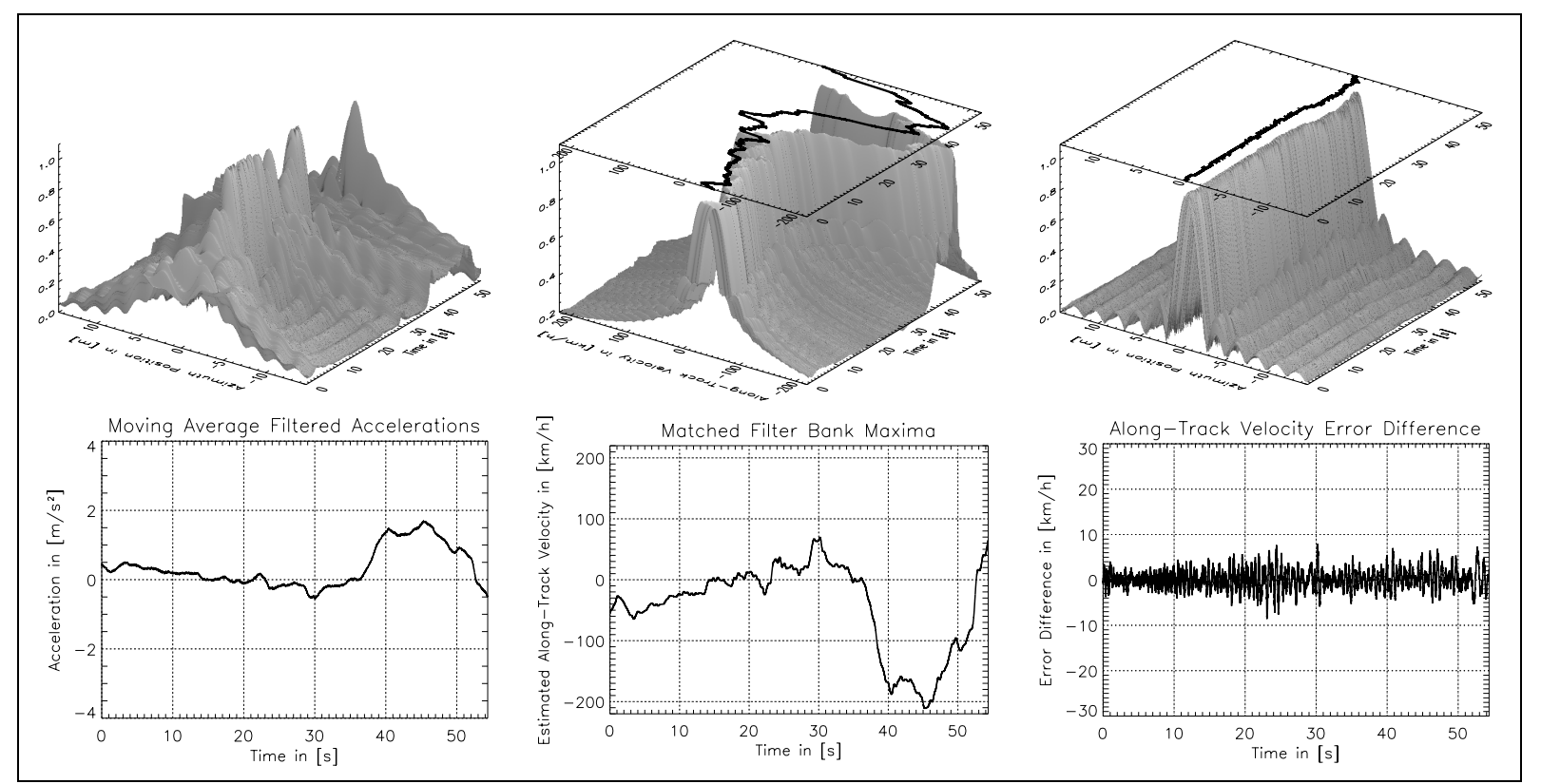

Figure 3: Example for estimating the along-track velocity error using measured acceleration data. The upper plots show the simulated profile of the azimuth impulse response (left, X-band), the profile of the matched filter bank response (middle) and the refocused azimuth impulse response. The lower plots show the moving average filtered accelerations (left), the estimated along-track velocity errors by matched filter bank (middle) and the difference between the estimated errors and the computed errors by using equation (2) (right).

\section{Vibration Measurements}

From the field of vehicle dynamics it is known that the eigen frequencies of the car body of a typical passenger car along the vertical axis lie between 0.7 and $2 \mathrm{~Hz}$ [3]. Indeed, significant vibration frequencies in this frequency range with amplitudes up to 3 centimetres were observable in the spectrogram of the measured vertical accelerations. The vibration amplitudes or car body deflections, respectively, depend mainly on the road unevenness [3]. To investigate the influence of vibrating point targets on SAR imagery simulations have been performed. Harmonic point target vibrations along the vertical axis were modelled by using for the z-coordinate of the target the time dependent expression $z=z(t)=A_{z} \cos \left(2 \pi f_{z} t+\varphi_{z}\right)$, where $A_{z}$ is the peak amplitude of the vibration, $f_{z}$ the frequency and $\varphi_{z}$ an initial phase shift.

In Figure 4 the simulated impulse responses of a vibrating point target with low frequency are shown. Especially at short integration times (e.g. at LEO systems) the shape of the impulse response is also influenced by the initial phase $\varphi_{z}$ since here the integration time is in the order of the vibration period. Beside the low frequencies of up to $2 \mathrm{~Hz}$ in the original (not filtered)
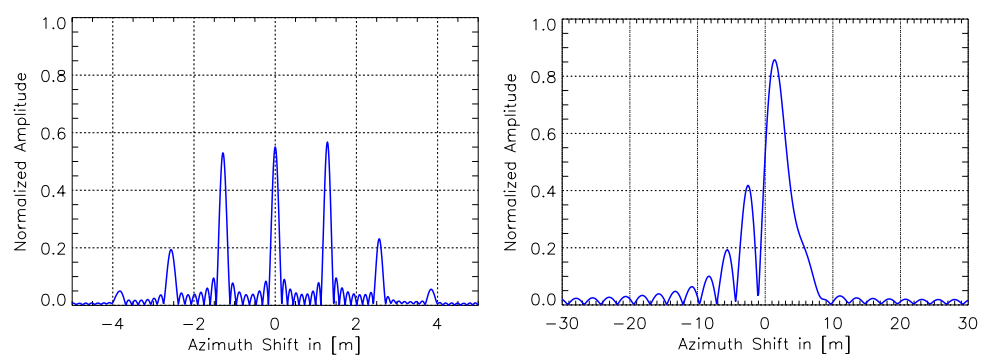

Figure 4: Impulse responses of a vibrating point target $\left(f_{z}=1.5 \mathrm{~Hz}\right.$, $A_{z}=5 \mathrm{~mm}, \varphi_{z}=-90^{\circ}$; left: airborne system, $T_{S A}=5.7 \mathrm{~s}$; right: LEO system, $T_{S A}=0.64 \mathrm{~s}$ ).

dataset also frequencies in the order of $10 \mathrm{~Hz}$ and $30 \mathrm{~Hz}$ were measured, but only with negligible small amplitudes. These frequencies can be assigned to wheel vibrations [3], to engine vibrations and to the internal control loop of the IMU. However, such high frequencies in combination with the small amplitudes have no significant influence on target's impulse response.

Although the influence on SAR imagery of vibrating point targets with low frequency clearly can be observed in the simulation results, these results cannot be transferred par to par to real vibrating vehicles, since no double bounce effects were simulated and since real vehicles are assumed to be not point like [2]. Further investigations and measurements are necessary before clear statements 
regarding vibrations of real vehicles can be made. Vibrations could potentially be exploited to gather information about road conditions.

\section{Reliable Along-Track Velocity Estimation}

The easiest way to separate across-track acceleration and along-track velocity, if no tracking should be performed, is the additional use of a road database. Once the across-track velocity and the azimuth
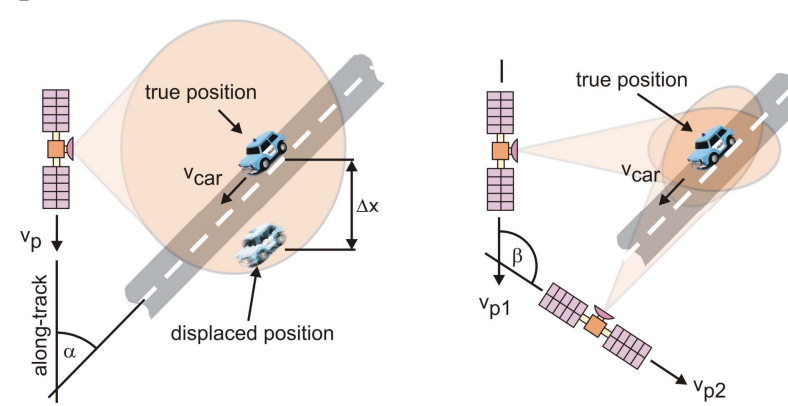

Figure 5: Possible configurations for resolving both ground velocity components (left: two- or multi-channel system integrated on a single platform; right: multistatic system).

displacement $\Delta x$ of the vehicle have been estimated, the vehicle can be assigned to a certain road, whereas the road direction $\alpha$ can be gained from a road database (Figure 5, left part). With the knowledge of the across-track velocity and the angle $\alpha$ the along-track velocity can be computed. The Doppler slope information now can be used for focusing the moving vehicle as well as for computing the across-track acceleration. However, the accuracy of the along-track velocity estimate depends strongly on the accuracy of the across-track velocity and on the magnitude of $\alpha$. Nevertheless, this method is inexpensive since no additional hardware is required and since for a traffic monitoring system anyhow a road database is necessary. Another method allowing the estimation of the velocity vector is the use of a multi-static system as shown in the right part of Figure 5. The beams of two or more radar satellites can be steered to a region of interest. Each satellite sees the vehicle moving with a different "across-track velocity", thus estimation of the vehicle velocity vector becomes feasible. Furthermore the detection probability is increased in contrast to a single-satellite system since most likely at least one "across-track velocity" exceeds the minimum-detectable velocity limit. Also a highly squinted geometry can be used where two satellites fly in a tandem configuration with large distance from each other (i.e. the angle $\beta$ in Figure 5 is increased to $180^{\circ}$ ).

\section{Conclusions}

The measurement results and simulations imply that acceleration standard deviations of up to $0.6 \mathrm{~m} / \mathrm{s}^{2}$ are not uncommon in real traffic scenarios. Using typical LEO systems even an unconsidered acrosstrack acceleration of $0.25 \mathrm{~m} / \mathrm{s}^{2}$ leads to an along-track velocity estimation error of about $-32 \mathrm{~km} / \mathrm{h}$. Having only a single- or two channel SAR system integrated on a single platform, additional information, e.g. from a road database, is needed to estimate the along-track velocity with high accuracy. The along-track velocity indeed is one important design driver for a radar based traffic monitoring system. Further investigations are necessary before such a system can be designed and realized.

\section{Reference:}

[1] J. Sharma et al., "The influence of target acceleration on velocity estimation in dual-channel SAR GMTI," IEEE Transactions on Geoscience and Remote Sensing, vol. 44, no. 1, January 2006.

[2] D. Hounam et al., "An autonomous, non-cooperative, wide-area Traffic Monitoring System using space-based Radar (TRAMRAD),“ IGARSS 2005, Seoul, Korea, July 2005.

[3] M. Mitschke, "Dynamik der Kraftfahrzeuge - Band B: Schwingungen," 2. Auflage, SpringerVerlag, 1984.

[4] S. Baumgartner et al., "Traffic Monitoring with SAR: Implications of Target Acceleration," EUSAR 2006, Dresden, Germany, May 2006. 